\title{
Pharmacokinetics and Safety of Amenamevir in Healthy Subjects: Analysis of Four Randomized Phase 1 Studies
}

\author{
Tomohiro Kusawake · James J. Keirns · Donna Kowalski • \\ Martin den Adel • Dorien Groenendaal-van de Meent • Akitsugu Takada • \\ Yoshiaki Ohtsu • Masataka Katashima
}

Received: September 20, 2017 / Published online: November 13, 2017

(C) The Author(s) 2017. This article is an open access publication

\begin{abstract}
Introduction: Amenamevir (ASP2151) is a nonnucleoside antiherpesvirus compound available for the treatment of varicella-zoster virus infections. In this article we summarize the findings of four phase 1 studies in healthy participants.

Methods: Four randomized phase 1 studies investigated the safety and pharmacokinetics of single and multiple doses of amenamevir, including the assessment of age group effect (nonelderly vs elderly), food effect, and the relative bioavailability of two formulations.
\end{abstract}

Enhanced content To view enhanced content for this article go to http://www.medengine.com/Redeem/ EFCCF0602464F533.

Electronic supplementary material The online

version of this article (https://doi.org/10.1007/s12325-

017-0642-4) contains supplementary material, which is available to authorized users.

T. Kusawake $(\varangle) \cdot$ J. J. Keirns · D. Kowalski Astellas Pharma Global Development Inc., Northbrook, IL, USA

e-mail: tomohiro.kusawake@astellas.com

M. den Adel · D. Groenendaal-van de Meent Astellas Pharma Europe B.V., Leiden, The Netherlands

A. Takada $\cdot$ M. Katashima Astellas Pharma Inc., Tokyo, Japan

Y. Ohtsu

Astellas Pharma Inc., Tsukuba, Japan
Amenamevir was administered orally at various doses as a single dose (5-2400 mg) or daily (300 or $600 \mathrm{mg} /$ day) for 7 days.

Results: Following single and multiple oral doses, amenamevir demonstrated a less than dose proportional increase in the pharmacokinetic parameters area under the plasma drug concentration versus time curve from time zero to infinity $\left(\mathrm{AUC}_{\mathrm{inf}}\right)$ and $C_{\text {max }}$. After single and multiple oral 300-mg doses of amenamevir, no apparent differences in pharmacokinetics were observed between nonelderly and elderly participants. In contrast, with the amenamevir 600 -mg dose both the area under the plasma drug concentration versus time curve from time zero to $24 \mathrm{~h}$ and $C_{\max }$ were slightly increased and renal clearance was decreased in elderly participants. The pharmacokinetics of amenamevir was affected by food, with $\mathrm{AUC}_{\text {inf }}$ increased by about $90 \%$. In the bioavailability study, $\mathrm{AUC}_{\text {inf }}$ and $C_{\text {max }}$ were slightly lower following tablet versus capsule administration (decreased by 14 and $12 \%$, respectively), with relative bioavailability of $86 \%$. The different amenamevir doses and formulations were safe and well tolerated; no deaths or serious adverse events were reported.

Conclusion: Amenamevir had less than dose proportional pharmacokinetic characteristics. Age may have an influence on amenamevir pharmacokinetics; however, the effect was considered minimal. The pharmacokinetics of amenamevir were affected by food, with $\mathrm{AUC}_{\mathrm{inf}}$ 
almost doubling when amenamevir was administered with food. The concentration versus time profile of the tablet was slightly lower than that of the capsule; the relative bioavailability of the tablet versus the capsule was $86 \%$. Amenamevir was safe and well tolerated in the dose range investigated.

Funding: Astellas Pharma.

Trial registration: ClinicalTrials.gov identifiers NCT02852876 (15L-CL-002) and NCT02796118 (15L-CL-003).

Keywords: Amenamevir; Japanese participants; Pharmacokinetics; Safety; Varicella-zoster virus

\section{INTRODUCTION}

Herpes zoster, or shingles, is the painful eruption of a rash caused by the varicella-zoster virus (VZV). Herpes zoster is more common in elderly people, patients with lymphoma, patients receiving chemotherapy or steroids, and people with HIV [1]. Antiviral therapy for herpes zoster is recommended in certain immunocompetent patients and all immunocompromised patients [2]. Nucleoside analogues such as acyclovir, valacyclovir, and famciclovir have been approved for the treatment of herpes simplex virus (HSV)-1, HSV-2, and VZV infections [3-5]. These drugs have the same mechanism of action and are widely used for the treatment of herpes zoster and herpes simplex.

Acyclovir-resistant virus has been isolated from patients with HSV. Acyclovir resistance is mediated by mutations in the thymidine kinase gene $[6,7]$ or the DNA polymerase gene [8]. Consequently, in clinical HSV isolates, resistance to acyclovir has been shown to be regularly linked to cross-resistance against penciclovir, whose prodrug is famciclovir [9-11]. Although acyclovir resistance of VZV is virtually unobserved in immunocompetent patients, it can be a major problem among immunosuppressed hosts, mainly those who have received prolonged acyclovir therapy [12-15].

A drug with a new mechanism of action with a lack of cross-resistance to current nucleoside analogues is desired. Amenamevir (ASP2151) is a nonnucleoside antiherpesvirus compound available for the treatment of VZV infections. Amenamevir targets the herpesvirus helicase-primase complex. This viral enzyme complex is the target for the next generation of antiherpes drugs [16, 17], as the helicase-primase complex of human Alphaherpesvirinae viruses is known to be essential for viral DNA replication and, hence, viral replication [16-20].

Preclinical pharmacology studies of amenamevir have demonstrated that amenamevir inhibits the replication of acyclovir-resistant strains of HSV-1, HSV-2, and VZV, with half-maximal effective concentrations similar to those exhibited against acyclovir-sensitive strains [21]. Initial pharmacokinetic results from two phase 1 studies (studies 15L-CL-002 and 15L-CL-003) have shown that amenamevir exhibits less than dose proportional pharmacokinetics after single and multiple doses [22]. Here we summarize the findings of four phase 1 studies (including further data from studies 15L-CL-002 and 15L-CL-003) to evaluate the safety and pharmacokinetics of single and multiple doses of amenamevir, including the assessment of age effect (nonelderly vs elderly), food effect, and the relative bioavailability of two formulations (capsule vs tablet).

\section{METHODS}

\section{Study Design, Participants, and Dosing}

The designs of the four phase 1 studies in healthy participants are included in the supplementary material (Table S1). The first study was a randomized, double-blind, placebo-controlled, dose-escalation study conducted in Japan (study 15L-CL-001). Sequential cohorts of healthy male participants (aged 20-44 years) were randomized to receive a single capsule dose of amenamevir or placebo under fasting conditions. The doses of amenamevir were 5, $25,100,300$, or $600 \mathrm{mg}$. This study was not registered at a registry website as there was no requirement in the International Council for Harmonisation of Technical Requirements for Pharmaceuticals for Human Use (ICH) 
"Guideline for Good Clinical Practice" to register phase 1 trials at the time it was conducted.

The second study, conducted in France (15L-CL-002; ClinicalTrials.gov identifier NCT02852876) in healthy male participants (aged 18-55 years), had two parts. Part 1 was a randomized, double-blind, placebo-controlled, single dose escalation study that evaluated the safety, tolerability, and pharmacokinetics of amenamevir 5-, 25-, 100-, 300-, 600-, 1200-, 1800-, and 2400-mg capsules under fasting conditions. Part 2 was an open-label crossover study that evaluated the effect of food on the safety, tolerability, and pharmacokinetics of a single capsule dose of $300 \mathrm{mg}$ amenamevir.

The third study was a randomized, double-blind, placebo-controlled, multiple ascending dose study conducted in Japan (15L-CL-003; ClinicalTrials.gov identifier NCT02796118). Healthy male nonelderly (aged 20-44 years) and elderly (aged 65-79 years) participants were randomized to receive amenamevir capsule, $300 \mathrm{mg} /$ day, or placebo capsules, or amenamevir capsule, $600 \mathrm{mg} /$ day, or placebo capsules, all administered under fed conditions for 7 days. The safety and pharmacokinetics of amenamevir were assessed over a 7-day period.

The final study was a randomized, open-label, three-period crossover study conducted in the USA (15L-CL-006). Healthy male and female participants received a single capsule dose of $800 \mathrm{mg}$ amenamevir under fasting conditions, a single tablet dose of $800 \mathrm{mg}$ amenamevir under fasting conditions, and a single tablet dose of $800 \mathrm{mg}$ amenamevir under fed conditions. Each participant underwent a 6-day washout between each period. The safety and pharmacokinetics of amenamevir were evaluated, including the relative bioavailability of amenamevir capsule and tablet formulations and the effect of food with the tablet formulation. This study was not registered at a registry website as there was no requirement in the $\mathrm{ICH}$ "Guideline for Good Clinical Practice" to register phase 1 trials at the time it was conducted.

The study protocols were approved by the institutional review boards at each study site (15L-CL-001 and 15L-CL-003 by OPHAC Hospital Institutional Review Board, Osaka, Japan; 15L-CL-002 by Comité Consultatif de
Protection des Personnes dans la Recherche Biomédicale, Hôpital Rovert Ballanger-Centre Daniel Eisenmann, Aulnay-sous-Bois, France; 15L-CL-006 by Independent Investigational Review Board, Plantation, FL, USA), and the studies were conducted in accordance with the ethical standards of the ICH "Guideline for Good Clinical Practice." All procedures followed were in accordance with the ethical standards of the responsible committee on human experimentation (institutional and national) and with the Helsinki Declaration of 1964, as revised in 2000. Written informed consent was obtained from all participants for their being included in these studies.

\section{Pharmacokinetic Assessments}

For all four studies, plasma and urine drug concentrations were determined by validated liquid chromatography-tandem mass spectrometry methods [22], and plasma drug concentration-time data were used to calculate the following pharmacokinetic parameters of amenamevir: peak drug concentration $\left(C_{\max }\right)$, half-life $\left(t_{1 / 2}\right)$, time to peak drug concentration $\left(t_{\text {max }}\right)$, and apparent oral clearance. For studies 15L-CL-001, 15L-CL-002, and 15L-CL-006, these parameters were all calculated after a single dose of amenamevir. For study 15L-CL-003, these parameters were assessed on days 1 and 7 . The area under the plasma drug concentration versus time curve from time zero to infinity $\left(\mathrm{AUC}_{\mathrm{inf}}\right)$ was also assessed in studies 15L-CL-001, 15L-CL-002, and 15L-CL-006, and the time that the amenamevir drug concentration was above $200 \mathrm{ng} / \mathrm{mL}\left(t_{200}\right)$ and the area under the plasma drug concentration versus time curve from time zero to $24 \mathrm{~h}\left(\mathrm{AUC}_{24}\right)$ were also assessed in study 15L-CL-003. Urine pharmacokinetics, including the cumulative amount of the unchanged drug excreted into the urine at $24 \mathrm{~h}$, the percentage of drug excreted in urine, and renal clearance were assessed in studies 15L-CL-003 and 15L-CL-006.

For study 15L-CL-001, fasting participants received amenamevir with $200 \mathrm{~mL}$ of water in the morning. Participants continued to refrain from having any food and drink until $4 \mathrm{~h}$ after 
dosing. Serial blood samples for amenamevir were collected before the dose and $0.25,0.5,1$, $1.5,2,3,4,6,8,10,12,24,36$, and $48 \mathrm{~h}$ after the dose.

For part 1 of study 15L-CL-002, fasting participants received amenamevir with $240 \mathrm{~mL}$ of water in the morning. Participants continued to refrain from having any food and drink until $4 \mathrm{~h}$ after dosing. For part 2 of study 15L-CL-002, amenamevir was administered either $30 \mathrm{~min}$ after an FDA high-fat breakfast or under the same fasting conditions as in part 1 of the study. Serial blood samples for amenamevir were collected before the dose and $0.25,0.5,1,1.5,2,3$, $4,5,6,7,8,10,12,16,24,36$, and $48 \mathrm{~h}$ after the dose.

For study 15L-CL-003, participants received amenamevir with $200 \mathrm{~mL}$ of water within $30 \mathrm{~min}$ after breakfast. Participants refrained from having any food and drink until $4 \mathrm{~h}$ after dosing. Serial blood samples for amenamevir were collected before the dose and $0.25,0.5,1$, $1.5,2,3,4,6,8,10$, and $12 \mathrm{~h}$ after the dose on day 1 , before the dose on day 2 to day 6 , and before the dose and $0.25,0.5,1,1.5,2,3,4,6,8$, $10,12,24,36$, and $48 \mathrm{~h}$ after the dose on day 7 . Urine samples for assessment of cumulative urinary excretion of amenamevir were collected at the following intervals: before the dose and 0-4 h, 4-8 h, 8-12 h, and 12-24 h after the dose on day 1 and before the dose and $0-4 \mathrm{~h}, 4-8 \mathrm{~h}$, 8-12 h, 12-24 h, 24-36 h, and 36-48 h after the dose on day 7 .

For study 15L-CL-006, participants who received their dose under fasting conditions received amenamevir with $240 \mathrm{~mL}$ of water. Participants continued to refrain from having any food and drink until $4 \mathrm{~h}$ after dosing. In participants who received their dose under fed conditions, amenamevir was administered 30 min after an FDA high-fat breakfast. Serial blood samples for amenamevir were collected before the dose and $0.25,0.5,1,1.5,2,3,4,6,8$, $10,12,16,24,36$, and $48 \mathrm{~h}$ after the dose. Urine samples for assessment of cumulative urinary excretion of amenamevir were collected at the following intervals: before the dose and $0-4 \mathrm{~h}$, $4-8 \mathrm{~h}, 8-12 \mathrm{~h}, 12-24 \mathrm{~h}, 24-36 \mathrm{~h}$, and $36-48 \mathrm{~h}$ after the dose.

\section{Safety Assessment}

In all four studies, safety was evaluated by our assessing treatment emergent adverse events, 12-lead electrocardiogram (ECG) recordings, clinical laboratory parameters (biochemistry, hematology, serology, and urinalysis), vital signs (blood pressure and pulse), and physical examination findings.

\section{Statistical Analysis}

Pharmacokinetic calculations were performed with actual sampling times by noncompartmental methods with use of WinNonlin ${ }^{\circledR}$ Professional version 5.0 or higher (Pharsight, Mountain View, CA, USA) or $\mathrm{SAS}^{\circledR}$ version 8.2 or higher (SAS Institute, Cary, NC, USA). In all four studies, descriptive statistics included the number of participants reflected in the calculation $(n)$, arithmetic mean, standard deviation, median, minimum, and maximum for continuous variables, and frequency and percentage for categorical end points.

For part 2 of study 15L-CL-002, the effect of food on the pharmacokinetics of the amenamevir capsule was assessed with the two one-sided $t$ test procedure. A mixed effects model with treatment, sequence, and period as fixed effects and participant as a random effect was used. The same two one-sided $t$ test procedure was used in study 15L-CL-003 to assess the effect of age on the pharmacokinetics of the amenamevir capsule and in study 15L-CL-006 to assess the relative bioavailability of the tablet and capsule formulations of amenamevir as well as the effect of food on the pharmacokinetics of amenamevir tablets. For study 15L-CL-003, a fixed effects model was used with age as an effect. For study 15L-CL-006, a mixed effects model with treatment, sequence, period, and sex as fixed effects and participant as a random effect was used. For all analyses within each model, the geometric least squares mean ratios (fed/fasting for assessing food effect; tablet/capsule for assessing relative bioavailability; elderly/ 
nonelderly for assessing an age group effect) and their corresponding 90\% confidence intervals for plasma $\mathrm{AUC}_{\mathrm{inf}}$ and $C_{\max }$ were obtained.

\section{RESULTS}

\section{Participants}

The baseline characteristics and demographics of the participants included in each study are reported in Table 1 . The participants enrolled in the four studies were generally comparable, with the exception that study 15L-CL-006 enrolled both male and female participants and study 15L-CL-003 included elderly participants and that studies 15L-CL-001 and 15L-CL-003 enrolled Japanese participants whereas studies 15L-CL-002 and 15L-CL-006 had mainly White participants. The flow of participants through each study is shown in Figs S1, S2, S3, and S4.

\section{Pharmacokinetics}

The mean concentration versus time curves following a single administration of amenamevir capsule by dose cohort in participants enrolled in study 15L-CL-001 and part 1 of study 15L-CL-002 are shown in Fig. 1. Furthermore, the pharmacokinetic parameter estimates for single-dose capsule administration of amenamevir in study 15L-CL-001 are presented in Table 2. These studies show that the mean $\mathrm{AUC}_{\text {inf }}$ and $C_{\max }$ increased in a less than dose proportional way, whereas the median $t_{\max }$ and mean $t_{1 / 2}$ were similar for each dose.

The geometric least squares mean ratios of elderly to nonelderly participants for $C_{\max }$ and $\mathrm{AUC}_{24}$ in study 15L-CL-003 are presented in Table 3. In participants receiving the amenamevir 300-mg dose, there were no consistent differences in the amenamevir pharmacokinetics between nonelderly and elderly participants. After a single 300-mg dose of amenamevir, elderly participants had a slight increase in

Table 1 Baseline characteristics

\begin{tabular}{|c|c|c|c|c|c|c|}
\hline & \multicolumn{6}{|l|}{ Study } \\
\hline & $\begin{array}{l}\text { 15L-CL-001 } \\
(n=40)\end{array}$ & $\begin{array}{l}\text { 15L-CL-002 } \\
\text { part } 1(n=64)\end{array}$ & $\begin{array}{l}\text { 15L-CL-002 } \\
\text { part } 2(n=8)\end{array}$ & $\begin{array}{l}\text { 15L-CL-003 } \\
\text { nonelderly } \\
(n=18)\end{array}$ & $\begin{array}{l}\text { 15L-CL-003 } \\
\text { elderly } \\
(n=18)\end{array}$ & $\begin{array}{l}\text { 15L-CL-006 } \\
(n=24)\end{array}$ \\
\hline Age (years) & $23.3 \pm 2.2$ & $33.1 \pm 9.2$ & $33.9 \pm 9.5$ & $23.3 \pm 4.8$ & $69.8 \pm 3.5$ & $40.0 \pm 10.7$ \\
\hline Male & $40(100 \%)$ & $64(100 \%)$ & $8(100 \%)$ & $18(100 \%)$ & $18(100 \%)$ & $12(50 \%)$ \\
\hline \multicolumn{7}{|l|}{ Race } \\
\hline White & 0 & $44(68.8 \%)$ & $7(87.5 \%)$ & 0 & 0 & $21(87.5 \%)$ \\
\hline $\begin{array}{l}\text { Black or } \\
\text { African } \\
\text { American }\end{array}$ & 0 & $15(23.4 \%)$ & 0 & 0 & 0 & $3(12.5 \%)$ \\
\hline Asian & $40(100 \%)$ & $2(3.1 \%)$ & 0 & $18(100 \%)$ & $18(100 \%)$ & 0 \\
\hline Other & 0 & $3(4.7 \%)$ & $1(12.5 \%)$ & 0 & 0 & 0 \\
\hline Weight (kg) & $62.6 \pm 8.0$ & $74.9 \pm 8.1$ & $76.0 \pm 12.7$ & $61.1 \pm 6.7$ & $63.3 \pm 7.7$ & $71.1 \pm 11.6$ \\
\hline Height (m) & $1.73 \pm 0.06$ & $1.77 \pm 0.06$ & $1.78 \pm 0.05$ & $1.74 \pm 0.05$ & $1.65 \pm 0.06$ & $1.66 \pm 0.11$ \\
\hline BMI $\left(\mathrm{kg} / \mathrm{m}^{2}\right)$ & $20.8 \pm 1.9$ & $24.0 \pm 2.2$ & $23.9 \pm 2.7$ & $20.2 \pm 1.9$ & $23.2 \pm 1.9$ & $25.6 \pm 2.5$ \\
\hline
\end{tabular}

All values are presented as the mean \pm standard deviation unless otherwise stated $B M I$ body mass index 

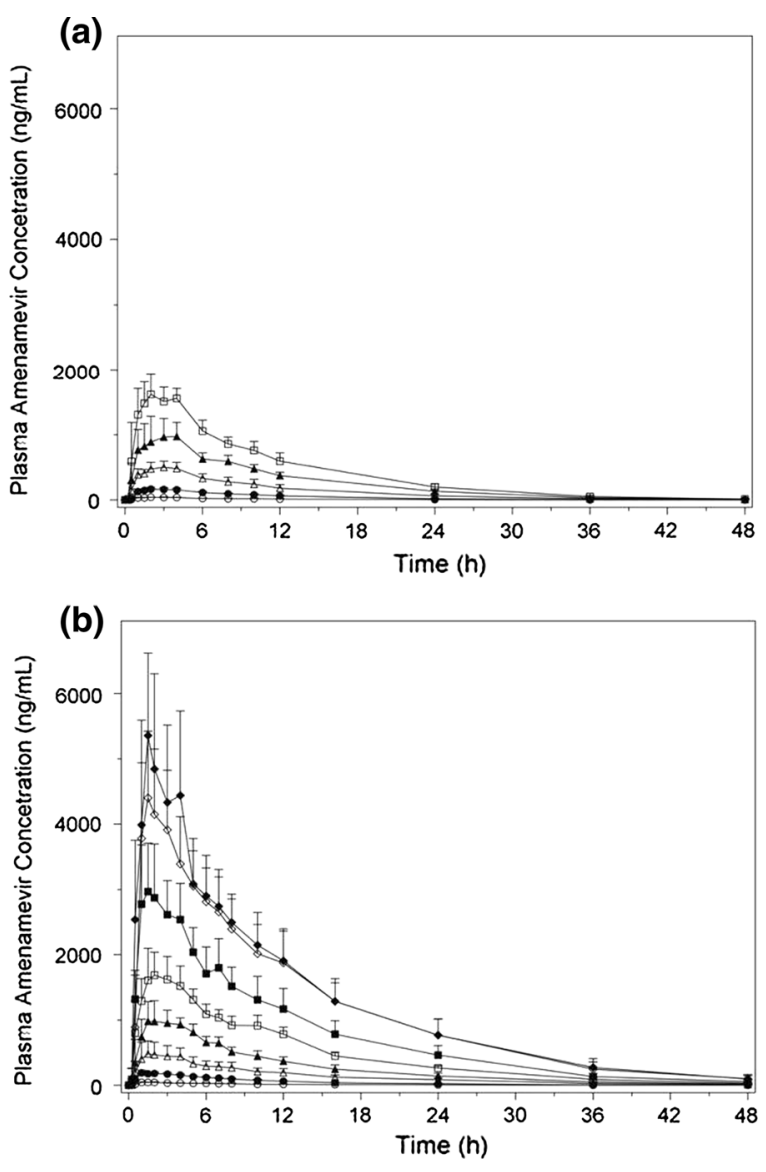

Fig. 1 Mean plasma concentration versus time profile of amenamevir in a study 15L-CL-001 and $\mathbf{b}$ part 1 of study 15L-CL-002 after a single capsule dose of amenamevir at $5 \mathrm{mg}$ (open circles), $25 \mathrm{mg}$ (closed circles), $100 \mathrm{mg}$ (open triangles), $300 \mathrm{mg}$ (closed triangles), $600 \mathrm{mg}$ (open squares), $1200 \mathrm{mg}$ (closed squares), $1800 \mathrm{mg}$ (open diamonds), and $2400 \mathrm{mg}$ (closed diamonds)

$\mathrm{AUC}_{24}$ and $C_{\text {max }}$ compared with nonelderly participants, whereas after multiple 300-mg doses of amenamevir, $\mathrm{AUC}_{24}$ and $C_{\max }$ were both slightly decreased in elderly participants. In contrast, in participants receiving amenamevir at $600 \mathrm{mg} /$ day, $C_{\max }$ and $\mathrm{AUC}_{24}$ of amenamevir were both slightly increased in elderly participants after single and multiple doses. In this study, the mean $t_{200}$ was around $24 \mathrm{~h}$ on day 7 for amenamevir at both $300 \mathrm{mg} /$ day and $600 \mathrm{mg} /$ day in both age groups (Table 4), which is assumed to be a relevant parameter for clinical efficacy [23].
In study 15L-CL-003, renal clearance of amenamevir ranged from 2.11 to $2.45 \mathrm{~L} / \mathrm{h}$ with $300 \mathrm{mg} /$ day and from 1.67 to $2.30 \mathrm{~L} / \mathrm{h}$ with $600 \mathrm{mg} /$ day. The percentage of drug excreted in urine was lower in participants receiving amenamevir at $600 \mathrm{mg} /$ day compared with participants receiving amenamevir at $300 \mathrm{mg} /$ day in both the nonelderly and the elderly participants. Renal clearance was lower in elderly participants compared with nonelderly participants after single and multiple doses of $600 \mathrm{mg}$ (Table 4).

The pharmacokinetics of amenamevir were affected by food. In part 2 of study 15L-CL-002, the $\mathrm{AUC}_{\mathrm{inf}}$ and $C_{\max }$ of a single capsule dose of $300 \mathrm{mg}$ amenamevir were increased when amenamevir was administered with food (Table 2, Fig. 2), $t_{1 / 2}$ remained the same, whereas apparent oral clearance was reduced. There was a $90 \%$ increase in $\mathrm{AUC}_{\text {inf }}$ and an $82 \%$ increase in $C_{\max }$ when amenamevir was administered with food relative to fasting conditions. Similar results were seen in study 15L-CL-006 (Table 5). Furthermore, in study 15L-CL-006, the percentage of amenamevir excreted unchanged in the urine in participants receiving the tablet formulation under fasting and fed conditions showed that the urine excretion increased when amenamevir was administered with food (Table 6).

The pharmacokinetics of the amenamevir tablet and capsule in fasting participants enrolled in study 15L-CL-006 are shown in Fig. 3 and Tables 5 and $6 . \mathrm{AUC}_{\text {inf }}$ and $C_{\max }$ were slightly lower following tablet versus capsule administration in the fasted state, which reflected a relative bioavailability of $86 \%$.

\section{Safety}

Amenamevir was safe and well tolerated. No deaths or serious adverse events were reported in any study. In studies 15L-CL-001, 15L-CL-002, and 15L-CL-003, all adverse events reported with amenamevir were mild in severity, and no adverse events resulted in participants discontinuing their participation in the study. Two participants discontinued their participation in study 15L-CL-006 because of an 
Table 2 Summary of amenamevir plasma pharmacokinetics after a single dose

\begin{tabular}{|c|c|c|c|c|c|c|c|}
\hline Study & $\begin{array}{l}\text { Amenamevir } \\
\text { dose (mg) }\end{array}$ & No. & $\begin{array}{l}\operatorname{AUC}_{\text {inf }} \\
(\mathbf{n g ~ h / m L})\end{array}$ & $C_{\max }(\mathrm{ng} / \mathrm{mL})$ & $t_{\max }(\mathbf{h})^{\mathbf{a}}$ & $t_{1 / 2}(\mathrm{~h})$ & $\begin{array}{l}\text { CL/F } \\
(\mathbf{L} / \mathbf{h})\end{array}$ \\
\hline \multirow[t]{6}{*}{ 15L-CL-001 } & 5 & 6 & $474 \pm 113$ & $42.9 \pm 3.99$ & $\begin{array}{l}1.75 \\
\quad(1.00-3.00)\end{array}$ & $7.75 \pm 1.58$ & $11.1 \pm 2.88$ \\
\hline & 25 & 6 & $1980 \pm 303$ & $177 \pm 21.9$ & $\begin{array}{l}2.50 \\
\quad(1.00-4.00)\end{array}$ & $7.42 \pm 0.59$ & $12.9 \pm 2.04$ \\
\hline & 100 & 6 & $5870 \pm 1670$ & $526 \pm 76.9$ & $\begin{array}{l}2.00 \\
\quad(1.00-3.00)\end{array}$ & $7.09 \pm 1.40$ & $18.4 \pm 5.71$ \\
\hline & 300 & 6 & $11,700 \pm 1920$ & $1040 \pm 264$ & $\begin{array}{l}3.00 \\
\quad(2.00-4.00)\end{array}$ & $6.88 \pm 0.56$ & $26.2 \pm 4.49$ \\
\hline & 600 & 6 & $18,700 \pm 2960$ & $1680 \pm 218$ & $\begin{array}{l}3.00 \\
\quad(2.00-4.00)\end{array}$ & $6.86 \pm 0.62$ & $32.7 \pm 4.78$ \\
\hline & $\begin{array}{l}\text { Dose } \\
\text { proportionality }\end{array}$ & - & $\begin{array}{l}0.766 \\
\quad(0.718-0.814)\end{array}$ & $\begin{array}{l}0.759 \\
\quad(0.720-0.799)\end{array}$ & - & - & - \\
\hline $\begin{array}{l}\text { 15-CL-002 } \\
\text { part } 1\end{array}$ & $\begin{array}{l}\text { Dose } \\
\quad \text { proportionality }\end{array}$ & - & $\begin{array}{l}0.750 \\
\quad(0.714-0.785)\end{array}$ & $\begin{array}{l}0.773 \\
\quad(0.740-0.806)\end{array}$ & - & - & - \\
\hline \multirow[t]{3}{*}{$\begin{array}{l}\text { 15-CL-002 } \\
\text { part } 2\end{array}$} & 300 (fasting) & 8 & $11,200 \pm 3420$ & $937 \pm 389$ & $\begin{array}{l}2.00 \\
\quad(1.5-5.0)\end{array}$ & $8.20 \pm 1.79$ & $29.2 \pm 9.8$ \\
\hline & $300(\mathrm{fed})$ & 8 & $20,700 \pm 4130$ & $1580 \pm 257$ & $\begin{array}{l}5.00 \\
\quad(3.0-5.0)\end{array}$ & $8.45 \pm 1.25$ & $15.0 \pm 2.8$ \\
\hline & $\mathrm{GMR}^{\mathrm{c}}$ & - & $\begin{array}{l}1.90 \\
(1.55-2.32)\end{array}$ & $\begin{array}{l}1.82 \\
\quad(1.34-2.48)\end{array}$ & - & - & - \\
\hline
\end{tabular}

All values are presented as the mean \pm standard deviation unless otherwise stated

$A U C_{\text {inf }}$ area under the plasma concentration versus time curve from time zero to infinity, $C L / F$ apparent oral clearance, $C_{\text {max }}$ peak drug concentration, GMR geometric least squares mean ratio (fed/fasting), $t_{1 / 2}$ terminal half-life, $t_{\max }$ time to peak drug concentration

a The median is presented (with the range in parentheses)

b Power model assessment of dose proportionality. The values presented are the slope (with the $95 \%$ confidence interval in parentheses)

c For the fed/fasting condition (with the $90 \%$ confidence interval in parentheses)

adverse event, although the events were not considered related to treatment.

In study 15L-CL-001, four of 30 participants $(13.3 \%)$ who received single doses of amenamevir experienced an adverse event: one participant each receiving amenamevir at a dose of $5,100,300$, or $600 \mathrm{mg}$. Only one adverse event was considered possibly related to the amenamevir dose of $300 \mathrm{mg}$ (increase in aspartate aminotransferase level).
In part 1 of study 15L-CL-002, four of 48 participants (8.3\%) who received single doses of amenamevir experienced an adverse event: one receiving $100 \mathrm{mg}$ amenamevir, one receiving $300 \mathrm{mg}$ amenamevir, and two receiving $600 \mathrm{mg}$ amenamevir. Of these, three events were considered related to study treatment: headache (unlikely), headache (possibly), and shoulder pain (possibly). No adverse events were reported in part 2 of study 15L-CL-002. 
Table 3 Geometric least squares mean ratio of elderly/ nonelderly participants for amenamevir plasma pharmacokinetics after multiple doses in study 15L-CL-003

\begin{tabular}{llll}
\hline Dose $(\mathbf{m g})$ & Day & $\mathrm{AUC}_{\mathbf{2 4}}(\mathbf{n g ~ h} / \mathbf{m L})$ & $\boldsymbol{C}_{\mathbf{m a x}}(\mathbf{n g} / \mathbf{m L})$ \\
\hline 300 & 1 & $1.09(0.85-1.38)$ & $1.05(0.81-1.35)$ \\
& 7 & $0.96(0.75-1.24)$ & $0.89(0.67-1.19)$ \\
600 & 1 & $1.28(0.92-1.77)$ & $1.18(0.93-1.50)$ \\
& 7 & $1.16(0.90-1.49)$ & $1.10(0.90-1.36)$ \\
\hline
\end{tabular}

The values presented are the geometric least squares mean ratio (with the $90 \%$ confidence interval in parentheses) for elderly/nonelderly participants

$A U C_{24}$ area under the plasma concentration versus time curve from time zero to $24 \mathrm{~h}, C_{\max }$ peak drug concentration

In study 15L-CL-003, adverse events were reported in four of six nonelderly participants (66.7\%) receiving $600 \mathrm{mg}$ amenamevir, three of six elderly participants (50.0\%) receiving placebo, and one elderly participant of six participants (16.7\%) receiving $600 \mathrm{mg}$ amenamevir. Five participants experienced an adverse event that was considered possibly related to treatment: two nonelderly participants receiving $600 \mathrm{mg}$ amenamevir (headache), one elderly participant receiving $600 \mathrm{mg}$ amenamevir (blood amylase level increased), and two elderly participants receiving placebo (headache and blood cholesterol level increased, respectively).

In study 15L-CL-006, 13 of 24 participants (54.2\%) reported 25 adverse events. The adverse events occurring in more than one participant were alopecia $(n=5)$, constipation $(n=3)$, upper respiratory tract infection $(n=2)$, and vomiting $(n=2)$. In all five cases of alopecia, the alopecia was mild in severity and occurred in female participants on day 15. It was described as finding hair on the pillow or increased hair loss on brushing. Examination of these participants did not reveal bald patches or scalp changes. Three adverse events were moderate in severity (pharyngitis, upper respiratory tract infection, and pain in an extremity; all $n=1$ ), and the remainder were mild.

In all four studies, there were no clinically significant changes from the baseline in vital signs, ECGs, or clinical laboratory assessments.

\section{DISCUSSION}

The helicase-primase inhibitor amenamevir offers an alternative therapeutic approach for patients with VZV infection. Data from the four human studies described here highlight the pharmacokinetics of amenamevir in various clinical settings (single and multiple dosing, food effect, age effect, and relative

Table 4 Summary of amenamevir urine pharmacokinetics and plasma $t_{200}$ after multiple doses in study 15L-CL-003

\begin{tabular}{|c|c|c|c|c|c|c|c|}
\hline Age group & Amenamevir dose (mg) & Day & No. & $\mathrm{Ae}_{24}(\mathrm{mg})$ & Ae\% & $\mathbf{C} \mathbf{L}_{\mathbf{R}}(\mathbf{L} / \mathbf{h})$ & $t_{200}(\mathrm{~h})$ \\
\hline \multirow[t]{4}{*}{ Nonelderly } & 300 & 1 & 6 & $33.22 \pm 9.50$ & $11.07 \pm 3.17$ & $2.45 \pm 0.53$ & $21.1 \pm 2.0$ \\
\hline & & 7 & 6 & $30.80 \pm 10.40$ & $10.27 \pm 3.47$ & $2.22 \pm 0.61$ & $21.5 \pm 2.9$ \\
\hline & 600 & 1 & 6 & $49.56 \pm 10.82$ & $8.26 \pm 1.80$ & $2.30 \pm 0.43$ & $22.8 \pm 0.5$ \\
\hline & & 7 & 6 & $42.13 \pm 12.84$ & $7.02 \pm 2.14$ & $2.15 \pm 0.64$ & $24.4 \pm 2.7$ \\
\hline \multirow[t]{4}{*}{ Elderly } & 300 & 1 & 6 & $30.06 \pm 3.77$ & $10.02 \pm 1.26$ & $2.11 \pm 0.53$ & $22.2 \pm 1.8$ \\
\hline & & 7 & 6 & $28.79 \pm 7.82$ & $9.60 \pm 2.61$ & $2.17 \pm 0.58$ & $23.8 \pm 4.8$ \\
\hline & 600 & 1 & 6 & $50.78 \pm 13.66$ & $8.46 \pm 2.28$ & $1.80 \pm 0.25$ & $22.9 \pm 1.1$ \\
\hline & & 7 & 6 & $39.30 \pm 13.72$ & $6.55 \pm 2.29$ & $1.67 \pm 0.43$ & $26.7 \pm 3.8$ \\
\hline
\end{tabular}

All values are presented as the mean \pm standard deviation

$\mathrm{Ae}_{24}$ cumulative amount of the unchanged drug excreted into the urine at $24 \mathrm{~h}, \mathrm{Ae} \%$ percentage of drug excreted in urine, $C L_{R}$ renal clearance, $t_{200}$ time above $200 \mathrm{ng} / \mathrm{mL}, t_{\max }$ time to peak drug concentration 


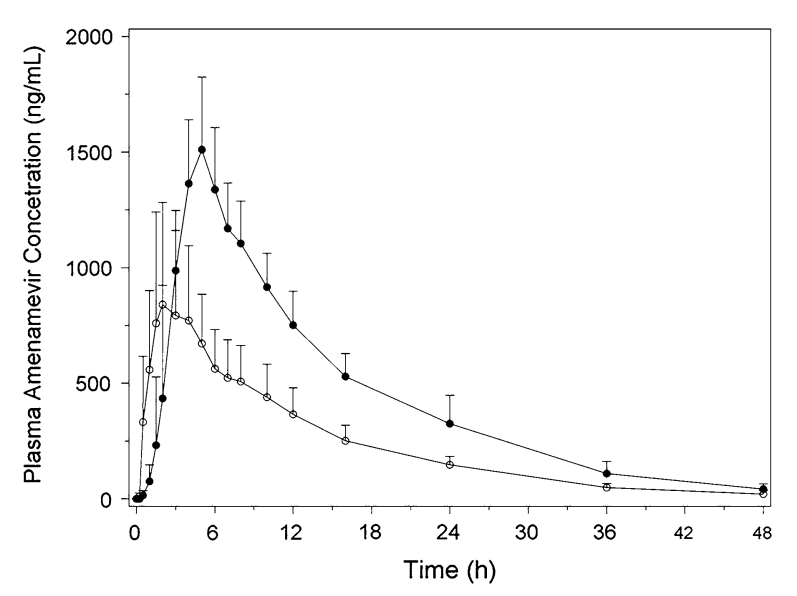

Fig. 2 Mean plasma concentration versus time profile of amenamevir after a single capsule dose under fasting (open circles) and fed (closed circles) conditions in part 2 of study 15L-CL-002

bioavailability of capsule versus tablet), as well as its safety and tolerability. Following single and multiple oral doses, amenamevir demonstrated a less than dose proportional increase in the pharmacokinetic parameters $\mathrm{AUC}_{\text {inf }}$ and $C_{\max }$. Amenamevir was rapidly absorbed, and $t_{\max }$ and $t_{1 / 2}$ of amenamevir were similar across all doses.

The pharmacokinetics of amenamevir were affected by food, with results showing that $\mathrm{AUC}_{\text {inf }}$ almost doubled when amenamevir was administered with food. $C_{\max }$ also increased with food (1.5-1.8-fold increase), and there was also a delay in $t_{\max }$ after eating. Food is known to affect the bioavailability of multiple oral drugs by various means, including delaying gastric emptying, stimulating bile flow, changing gastrointestinal $\mathrm{pH}$, increasing splanchnic blood flow, changing luminal metabolism of a drug substance, and physically or chemically interacting with a dosage form or a drug substance [24]. Although the cause of the changes in the pharmacokinetics of amenamevir with food is unknown, it is likely due to the low solubility of amenamevir. After food is consumed, bile acid is released. Bile acid is likely to increase the solubility of amenamevir and, therefore, the increase in exposure to amenamevir with food may be due to the increased levels of bile acid. In any case, the effect of food on the pharmacokinetics of amenamevir should be taken into consideration when one is selecting the appropriate time of the day to administer amenamevir as well as the appropriate dose.

Age may have a minimal influence on the pharmacokinetics of amenamevir, as demonstrated in study 15L-CL-003. In this study, in participants receiving the amenamevir 300-mg dose, there were no consistent differences in amenamevir pharmacokinetics between nonelderly and elderly participants. In participants receiving amenamevir at $600 \mathrm{mg} /$ day, $C_{\max }$ and $\mathrm{AUC}_{24}$ of amenamevir were both slightly increased and renal clearance was decreased in elderly participants after single and multiple doses. However, the $90 \%$ confidence interval of the geometric least squares mean ratios for both pharmacokinetic parameters across the dose range was wide and included 1 ; therefore, the age effect was considered to be minimal.

Two of the studies described here were conducted in Japanese participants and two were conducted in predominantly White populations. Although a direct comparison was not possible across the separate studies, the pharmacokinetic parameters of amenamevir in the two single ascending dose studies were similar [22], suggesting that there were no substantial differences between Japanese and non-Japanese participants.

In study 15L-CL-006, there were slight differences in the pharmacokinetics of the amenamevir tablet and capsule in fasting male and female participants. In this study, $\mathrm{AUC}_{\text {inf }}$ and $C_{\max }$ were slightly lower following tablet versus capsule administration in the fasted state, and this is reflected by a relative bioavailability of $86 \%$. Subsequent studies of amenamevir used the tablet formulation as the final product.

In study 15L-CL-003, the mean $t_{200}$ of amenamevir on day 7 was around $24 \mathrm{~h}$ (range 21.5-26.7 h) for all doses investigated. In a murine pharmacokinetic/pharmacodynamic analysis of amenamevir, it was determined that $t_{100}$, the length of time the amenamevir concentration in plasma exceeds $100 \mathrm{ng} / \mathrm{mL}$, was the most reasonable way to predict the efficacy of amenamevir with respect to the complete inhibition of HSV-1 replication [23]. As the activity of amenamevir against VZV is 
Table 5 Summary of amenamevir capsule and tablet plasma pharmacokinetics after a single dose in study 15L-CL-006

\begin{tabular}{|c|c|c|c|c|c|c|c|c|}
\hline Formulation & Condition & Sex & No. & $\begin{array}{l}\mathrm{AUC}_{\text {inf }} \\
(\mathrm{ng} \mathrm{h} / \mathrm{mL})\end{array}$ & $\begin{array}{l}\mathrm{C}_{\max }(\mathrm{ng} / \\
\mathrm{mL})\end{array}$ & $t_{\max }(\mathbf{h})^{\mathbf{a}}$ & $t_{1 / 2}(\mathbf{h})$ & $\begin{array}{l}\text { CL/F } \\
(\mathbf{L} / \mathbf{h})\end{array}$ \\
\hline \multirow[t]{3}{*}{ Capsule } & Fasting & Male & 12 & $26,900 \pm 8800$ & $2050 \pm 713$ & $2.0(2.0-4.0)$ & $7.8 \pm 1.1$ & $32.2 \pm 8.5$ \\
\hline & & Female & 12 & $31,800 \pm 9340$ & $2720 \pm 721$ & $2.0(1.5-4.0)$ & $7.2 \pm 1.1$ & $27.2 \pm 7.8$ \\
\hline & & $\begin{array}{c}\text { Male and } \\
\text { female }\end{array}$ & 24 & $29,300 \pm 9220$ & $2390 \pm 779$ & $2.0(1.5-4.0)$ & $7.5 \pm 1.2$ & $29.7 \pm 8.4$ \\
\hline \multirow[t]{6}{*}{ Tablet } & Fasting & Male & 12 & $23,700 \pm 10,400$ & $1900 \pm 851$ & $2.0(1.0-6.0)$ & $7.9 \pm 1.0$ & $40.7 \pm 19.9$ \\
\hline & & Female & 12 & $30,200 \pm 15,600$ & $2570 \pm 1060$ & $2.0(1.5-8.0)$ & $7.1 \pm 0.8$ & $34.1 \pm 18.8$ \\
\hline & & $\begin{array}{l}\text { Male and } \\
\text { female }\end{array}$ & 24 & $27,000 \pm 13,400$ & $2230 \pm 998$ & $2.0(1.0-8.0)$ & $7.5 \pm 1.0$ & $37.4 \pm 19.2$ \\
\hline & Fed & Male & 10 & $45,760 \pm 11,914$ & $3000 \pm 501$ & $4.0(4.0-8.0)$ & $7.9 \pm 1.2$ & $18.7 \pm 5.6$ \\
\hline & & Female & 12 & $50,573 \pm 15,170$ & $3420 \pm 662$ & $4.0(3.0-8.0)$ & $7.0 \pm 1.1$ & $17.0 \pm 4.7$ \\
\hline & & $\begin{array}{l}\text { Male and } \\
\text { female }\end{array}$ & 22 & $48,400 \pm 13,700$ & $3230 \pm 619$ & $4.0(3.0-8.0)$ & $7.4 \pm 1.2$ & $17.8 \pm 5.1$ \\
\hline \multicolumn{3}{|c|}{ GMR capsule/tablet ${ }^{\mathrm{b}}$} & - & $0.86(0.77-0.95)$ & $\begin{array}{l}0.88 \\
\quad(0.78-1.00)\end{array}$ & - & - & - \\
\hline \multicolumn{3}{|c|}{ GMR tablet fed/fasting ${ }^{\mathrm{b}}$} & - & $1.92(1.67-2.22)$ & $\begin{array}{l}1.55 \\
\quad(1.34-1.81)\end{array}$ & - & - & - \\
\hline
\end{tabular}

Values are presented as the mean \pm standard deviation unless otherwise stated

$A U C_{i n f}$ area under the plasma concentration versus time curve from time zero to infinity, $C L / F$ apparent oral clearance, $C_{\text {max }}$ peak drug concentration, $G M R$ geometric least squares mean ratio, $t_{1 / 2}$ terminal half-life, $t_{\text {max }}$ time to peak drug concentration

a The median is presented (with the range in parentheses)

b The $90 \%$ confidence interval is given in parentheses

Table 6 Summary of amenamevir urine pharmacokinetics in study 15L-CL-006

\begin{tabular}{llllll}
\hline Formulation & Condition & No. & $\mathbf{A e}_{\text {last }}(\mathbf{m g})$ & $\mathbf{A e} \%$ & $\mathbf{C L}_{\mathbf{R}}(\mathbf{L} / \mathbf{h})$ \\
\hline Capsule & Fasting & 24 & $54.89 \pm 14.05$ & $6.86 \pm 1.76$ & $2.00 \pm 0.56$ \\
Tablet & Fasting & 24 & $49.08 \pm 20.57$ & $6.13 \pm 2.57$ & $1.94 \pm 0.49$ \\
& Fed & 22 & $86.64 \pm 20.04$ & $10.83 \pm 2.51$ & $1.93 \pm 0.60$ \\
\hline
\end{tabular}

All values are presented as the mean \pm standard deviation

$A e_{\text {last }}$ cumulative amount of the unchanged drug excreted into the urine, $A e \%$ percentage of drug excreted in urine, $C L_{R}$ renal clearance

approximately twofold lower than that against HSV-1 [23], it was speculated that VZV growth can be suppressed with a dosage regimen resulting in a $t_{200}$ of close to $24 \mathrm{~h}$ per day. As such, the results of study 15L-CL-003 suggest that amenamevir may be effective against VZV.
Amenamevir was safe and well tolerated across all doses investigated in these studies. No deaths or serious adverse events were reported, and no clinically significant effects on vital signs, ECG, and clinical laboratory parameters were observed. These results are consistent with 


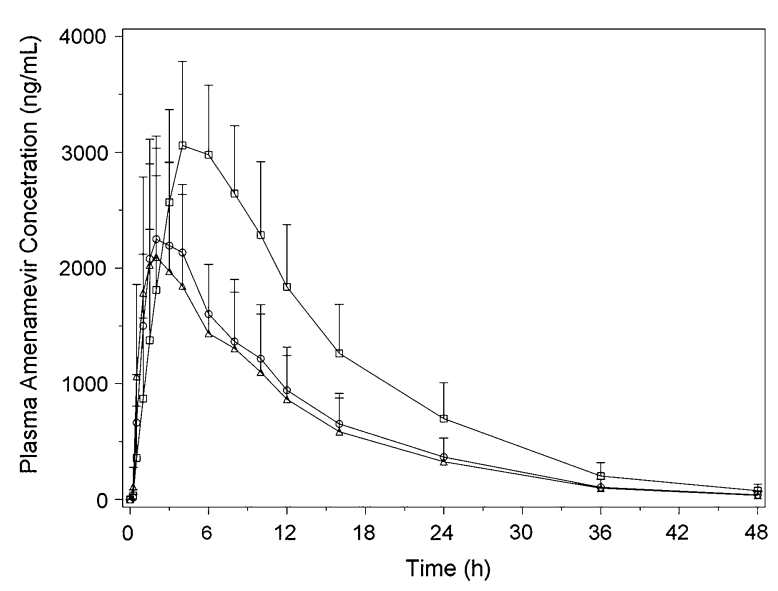

Fig. 3 Mean plasma concentration versus time profiles of amenamevir after a single capsule (open circles) or tablet (open triangles) dose under fasting conditions as well as after a single tablet dose under fed conditions (open squares) in study 15L-CL-006

the phase 2 clinical study of amenamevir in patients with recurrent genital herpes [25].

Like most phase 1 studies, the sample sizes were relatively small, which is a limitation of these studies and may affect the interpretation of the results.

\section{CONCLUSION}

In conclusion, amenamevir was safe and well tolerated at single (up to $2400 \mathrm{mg}$ ) and multiple (up to $600 \mathrm{mg} /$ day for 7 days) doses and showed less than dose proportional pharmacokinetic characteristics.

\section{ACKNOWLEDGEMENTS}

Sponsorship for the studies, article processing charges, and the open access fee were funded by Astellas Pharma. All authors had full access to all of the data in these studies and take complete responsibility for the integrity of the data and accuracy of the data analysis. We thank Simone Boniface of Springer Healthcare Communications, who wrote the first draft of the manuscript. This medical writing assistance was funded by Astellas Pharma. All named authors meet the International Committee of Medical Journal Editors (ICMJE) criteria for authorship for this manuscript, take responsibility for the integrity of the work as a whole, and have given final approval to the version to be published.

Disclosures. Tomohiro Kusawake is an employee of Astellas Pharma. Donna Kowalski is an employee of Astellas Pharma. Martin den Adel is an employee of Astellas Pharma. Dorien Groenendaal-van de Meent is an employee of Astellas Pharma. Akitsugu Takada is an employee of Astellas Pharma. Yoshiaki Ohtsu is an employee of Astellas Pharma. Masataka Katashima is an employee of Astellas Pharma. James J. Keirns was an employee of Astellas Pharma at the time these studies were conducted and retired from Astellas Pharma in April 2017. James J. Keirns is now an independent consultant in clinical pharmacology. The authors have no other relevant affiliations or financial involvement with any organization or entity with a financial interest in, or a financial conflict with, the subject matter or materials discussed in the manuscript other than those disclosed.

Compliance with Ethics Guidelines. The study protocols were approved by the institutional review boards at each study site (15L-CL-001 and 15L-CL-003 by OPHAC Hospital Institutional Review Board, Osaka, Japan; 15L-CL-002 by Comité Consultatif de Protection des Personnes dans la Recherche Biomédicale, Hôpital Rovert Ballanger-Centre Daniel Eisenmann, Aulnay-sous-Bois, France; 15L-CL-006 by Independent Investigational Review Board, Plantation, FL, USA), and the studies were conducted in accordance with the ethical standards of the International Council for Harmonisation of Technical Requirements for Pharmaceuticals for Human Use "Guideline for Good Clinical Practice." All procedures followed were in accordance with the ethical standards of the responsible committee on human experimentation (institutional and national) and with the Helsinki Declaration of 1964, as revised in 2000. Written informed consent was obtained from all participants for their being included in these studies. 
Data Availability. The data sets generated during and/or analyzed during the current study are not publicly available because these studies are small single-center studies and anonymization of the data is difficult to achieve.

Open Access. This article is distributed under the terms of the Creative Commons Attribution-NonCommercial 4.0 International License (http://creativecommons.org/licenses/ by-nc/4.0/), which permits any noncommercial use, distribution, and reproduction in any medium, provided you give appropriate credit to the original author(s) and the source, provide a link to the Creative Commons license, and indicate if changes were made.

\section{REFERENCES}

1. Wareham DW, Breuer J. Herpes zoster. BMJ. 2007;334(7605):1211-5.

2. Cohen JI. Herpes zoster. N Engl J Med. 2013;369(18):1766-7.

3. Perry CM, Faulds D. Valaciclovir. A review of its antiviral activity, pharmacokinetic properties and therapeutic efficacy in herpesvirus infections. Drugs. 1996;52(5):754-72.

4. Perry CM, Wagstaff AJ. Famciclovir. A review of its pharmacological properties and therapeutic efficacy in herpesvirus infections. Drugs. 1995;50(2):396-415.

5. Wagstaff AJ, Faulds D, Goa KL. Aciclovir. A reappraisal of its antiviral activity, pharmacokinetic properties and therapeutic efficacy. Drugs. 1994;47(1):153-205.

6. Bestman-Smith J, Schmit I, Papadopoulou B, Boivin G. Highly reliable heterologous system for evaluating resistance of clinical herpes simplex virus isolates to nucleoside analogues. J Virol. 2001;75(7):3105-10.

7. Morfin F, Thouvenot D. Herpes simplex virus resistance to antiviral drugs. J Clin Virol. 2003;26(1):29-37.

8. Larder BA, Darby G. Selection and characterisation of acyclovir-resistant herpes simplex virus type 1 mutants inducing altered DNA polymerase activities. Virology. 1985;146(2):262-71.
9. Sarisky RT, Quail MR, Clark PE, et al. Characterization of herpes simplex viruses selected in culture for resistance to penciclovir or acyclovir. J Virol. 2001;75(4):1761-9.

10. Sauerbrei A, Bohn K, Heim A, et al. Novel resistance-associated mutations of thymidine kinase and DNA polymerase genes of herpes simplex virus type 1 and type 2. Antivir Ther. 2011;16(8):1297-308.

11. Sauerbrei A, Deinhardt S, Zell R, Wutzler P. Phenotypic and genotypic characterization of acyclovir-resistant clinical isolates of herpes simplex virus. Antiviral Res. 2010;86(3):246-52.

12. Jacobson MA, Berger TG, Fikrig S, et al. Acyclovir-resistant varicella zoster virus infection after chronic oral acyclovir therapy in patients with the acquired immunodeficiency syndrome (AIDS). Ann Intern Med. 1990;112(3):187-91.

13. Linnemann CC Jr, Biron KK, Hoppenjans WG, Solinger AM. Emergence of acyclovir-resistant varicella zoster virus in an AIDS patient on prolonged acyclovir therapy. AIDS. 1990;4(6):577-9.

14. Pahwa S, Biron K, Lim W, et al. Continuous varicella-zoster infection associated with acyclovir resistance in a child with AIDS. JAMA. 1988;260(19):2879-82.

15. Snoeck R, Gérard M, Sadzot-Delvaux C, et al. Meningoradiculoneuritis due to acyclovir-resistant varicella zoster virus in an acquired immune deficiency syndrome patient. J Med Virol. 1994;42(4):338-47.

16. Crute JJ, Grygon CA, Hargrave KD, et al. Herpes simplex virus helicase-primase inhibitors are active in animal models of human disease. Nat Med. 2002;8(4):386-91.

17. Kleymann G, Fischer R, Betz UA, et al. New helicase-primase inhibitors as drug candidates for the treatment of herpes simplex disease. Nat Med. 2002;8(4):392-8.

18. Crute JJ, Mocarski ES, Lehman IR. A DNA helicase induced by herpes simplex virus type 1 . Nucleic Acids Res. 1988;16(14A):6585-96.

19. Crute JJ, Tsurumi T, Zhu LA, et al. Herpes simplex virus 1 helicase-primase: a complex of three herpes-encoded gene products. Proc Natl Acad Sci U S A. $1989 ; 86(7): 2186-9$.

20. Dodson MS, Crute JJ, Bruckner RC, Lehman IR. Overexpression and assembly of the herpes simplex virus type 1 helicase-primase in insect cells. J Biol Chem. 1989;264(35):20835-8. 
21. Chono K, Katsumata K, Suzuki H, Shiraki K. Synergistic activity of amenamevir (ASP2151) with nucleoside analogs against herpes simplex virus types 1 and 2 and varicella-zoster virus. Antiviral Res. 2013;97(2):154-60.

22. Ohtsu Y, van Trigt R, Takama K, et al. Quantification of ASP2151 in human plasma and urine: a pitfall associated with supersaturation of analyte in urine. Chromatographia. 2017;80(2):217-27.

23. Katsumata K, Chono K, Kato K, et al. Pharmacokinetics and pharmacodynamics of ASP2151, a helicase-primase inhibitor, in a murine model of herpes simplex virus infection. Antimicrob Agents Chemother. 2013;57(3):1339-46.

24. US Department of Health and Human Services. Guidance for industry: food-effect bioavailability and fed bioequivalence studies. 2002. https://www. fda.gov/downloads/regulatoryinformation/guid ances/ucm126833.pdf. Accessed 27 Jun 2017

25. Tyring S, Wald A, Zadeikis N, Dhadda S, Takenouchi K, Rorig R. ASP2151 for the treatment of genital herpes: a randomized, double-blind, placebo- and valacyclovir-controlled, dose-finding study. J Infect Dis. 2012;205(7):1100-10. 